\title{
L'entrepreneuriat innovant et le développement du secteur audiovisuel au Maroc. Cas de la Société Nationale de Radiodiffusion et de Télévision
}

\author{
Mohammed BELBACHIR $^{1}$ and Rachid ZAMMAR ${ }^{2}$ \\ ${ }^{I}$ Laboratoire d'études et de recherches en sciences de gestion, Université Mohammed V, Rabat, Maroc \\ ${ }^{2}$ Laboratoire d'études et de recherches en sciences de gestion, Université Mohammed V, Rabat, Maroc
}

\begin{abstract}
Résumé : Depuis l'émergence de la pandémie Covid 19, plusieurs secteurs dans le monde ont été véritablement impactés. Ainsi, le secteur audiovisuel au Maroc a beaucoup souffert de cette conjoncture. Le recours à l'entrepreneuriat innovant devient une nécessité incontournable. Pour cette raison, le gouvernement marocain a instauré une panoplie de mesures d'ordre financier, juridique et administratif afin de relancer ce secteur. Dans cette optique, nous ambitionnons à travers cet article de mettre en relief l'interaction entre l'entrepreneuriat innovant et le secteur audiovisuel au Maroc.

Ainsi, ce papier vise à décortiquer à quelle ampleur l'entrepreneuriat innovant peut impacter le secteur audiovisuel ainsi que les multiples programmes établis afin de promouvoir ce secteur. Afin de bien formuler une vision plus claire et exhaustive, notre étude a été focalisée sur la société Nationale de Radiodiffusion et de Télévision (SNRT) étant la locomotive de ce secteur au Maroc. Pour ce faire, une étude documentaire a été également réalisée par le biais des développements scientifiques et des analyses de monographies, des rapports et des graphiques portant sur ce domaine surtout de cette société. Cette analyse documentaire nous a permis de confirmer l'importance de l'entrepreneuriat étant un facteur névralgique de la relance de la dynamique de ce secteur à court terme. Cependant, vu le coût élevé généré par l'introduction de nouveaux procédés et technologies, l'impact de l'innovation se sera concrétisé qu'après une période bien déterminée.
\end{abstract}

Mots-clés : Entrepreneuriat, Innovation, Audiovisuel, Maroc.

\section{INTRODUCTION}

Le secteur d'audiovisuel au Maroc a connu une évolution majeure depuis la délivrance du Dahir ${ }^{1} \mathrm{n}^{\circ} 1-2$ 212 le 31 août 2002. Cette phase avancée s'est avérée décisive. Ainsi, elle a permis d'instaurer un cadre juridique ayant visé à libérer le secteur du monopole public. En outre, ce développement a identifié la structure organisationnelle et le cadre de l'intervention du secteur public ainsi que les conditions légales qui permettent l'accès des acteurs privés au secteur.

Par ailleurs, le Maroc a poursuivi sa stratégie de rationalisation et de renforcement de secteur en soutenant les différentes parties prenantes, en modernisant les plateformes de diffusion et en accompagnant les grands chantiers d'innovation et de développement de secteur audiovisuel national. En plus, il a fourni assez d'efforts afin d'améliorer la qualité des services offerts dans un environnement marqué par la libération continue.

\footnotetext{
${ }^{1}$-https://www.haca.ma/fr/st\%C3\%A9-de-laudiovisuel-public-sap
} 
En dépit des ressources matérielles, financières et humaines déployées par le gouvernement marocain, ainsi que les néfastes percussions économiques et sociales sur la croissance économique du pays et surtout le secteur audiovisuel ce secteur souffre d'un certain nombre de limites. D'où le recours à l'entrepreneuriat innovant devient une nécessité déterminante dans le but de relancer ce secteur et le rendre plus compétitif et rentable.

L'entrepreneuriat innovant est dû à la relation entre des individus et des opportunités (Shane 2003). Il est donc la coalition de deux concepts tels que l'entrepreneuriat et l'innovation. Il peut être considéré comme les deux faces d'une même pièce (Soriano et Huarng 2013).

Ainsi, l'entrepreneuriat innovant constitue un vecteur vital pour amortir les effets de l'impact de la récession économique à l'ère de la pandémie Covid 19. Dans ce contexte et suite à l'élaboration d'un nouveau modèle de développement économique, notre question principale autour de laquelle est axée notre recherche est : Dans quelle mesure l'entrepreneuriat innovant peut impacter la relance et le développement du secteur d'audiovisuel au Maroc?

Dans le but de bien analyser toutes les facettes de notre sujet, dans un premier temps, nous avons réalisé une revue des multiples références académiques portant sur les concepts de l'entrepreneuriat, l'innovation et les traits des travaux de recherches entre l'entrepreneuriat innovant et le secteur audiovisuel. Dans un deuxième temps, nous avons présenté la méthodologie adoptée lors de cette étude. Il s'agit d'une étude documentaire descriptive de différents rapports, diagrammes, schémas et résultats. Pour finir, une troisième section a été réservée à l'interprétation et la discutions des résultats obtenus.

\section{REVUE DE LITTERATURE}

\section{A. L'entrepreneuriat innovant}

L'entrepreneuriat est un concept hétérogène et complexe (Verstraete, 2001). Plusieurs chercheurs ont évoqué aussi la notion temporelle dans l'identification de ce concept comme (Fisher et al. 2016). Quant à Landström (2007), il a confirmé qu'il existe plusieurs définitions.

Il est le domaine qui ne cesse d'évoluer au fil du temps. Selon (Robert Heinrich et Micheal Peters, 2018), l'entrepreneuriat est considéré comme le processus de la création de la nouveauté en rationnalisant le temps et les ressources déployés. Ainsi, il permet de mettre en face toute sorte de risque et de récolter le profit escompté et la satisfaction personnelle (Fauzi and Soehari, 2020)

En outre, il est reconnu comme un facteur crucial du développement économique dans le monde entier (Erken et al. 2018 ; Urbano et Aparício 2016). Il stimule la croissance économique en introduisant l'innovation, en favorisant la concurrence et en augmentant la compétitivité (Vivarelli 2013 ; Wong et al. 2005).

Par ailleurs, un entrepreneur est un décideur vigilant qui cherche à décortiquer des opportunités (Kirzner 1973). C'est un entrepreneur qui perçoit une opportunité et crée une nouvelle (Bygrave, 1992). Autres paramètres tels que les attributs culturels et la formation peuvent impacter les comportements entrepreneuriaux (Knight, 1997 ; Kreiser, 1997). Des contributions empiriques ont approuvé ce constat (McGrath et al. 1992) et (Mueller et Thomas(2001). 
Or, la conjoncture mondiale devient de plus en plus instable. L'accroissement de la potentialité de la compétitivité demeure une nécessité irréversible. D'où le recours à l'innovation est devenu incontournable dans la vie des organisations.

En effet, d'après (Doloreux et Melancon, 2008 ; Hua et Wemmerlov, 2006 ; Roberts et Amit, 2003) l'innovation est un facteur décisif pour l'entreprise afin de s'adapter aux exigences d'un environnement turbulent. Ainsi, elle permet à une telle unité de bien se positionner dans un environnement instable et par conséquent augmenter son profit (Hu et Hsu, 2008 ; Kaminski et al. 2008).

Miller (1983) a proposé une interprétation plus large du concept de l'innovation qui a suscité la plupart des études. Lors du processus de l'innovation, des récentes technologies évoluées, efficaces et issues de la recherche scientifique sont utilisées pour minimiser les charges et par conséquent permettre aux entreprises de bien se localiser dans un marché compétitif (Baregheh et al. 2009). Il parait aussi que l'innovation permet d'accroître davantage la compétitivité et le niveau de la performance dans une organisation à travers son impact positif sur la connaissance (Koc et Ceylan, 2007).

En plus, l'innovation peut être considérée comme un outil que l'entrepreneur utilise pour stimuler le développement de son organisme. C'est un instrument essentiel pour faciliter la croissance de l'entreprise (Akinwale et al. 2018 ; Holcombe 1998 ; Drucker 1985).

\section{B. L'entrepreneuriat innovant et le secteur audiovisuel}

Le monde entier a connu une nette évolution dans plusieurs domaines, d'où l'apparition de nouvelles formes de l'entrepreneuriat. Or, Drucker (1985) a mis la lumière sur la relation entre l'entrepreneuriat et l'innovation. En effet, selon l'approche culturelle contemporaine, cette interaction est centrée sur l'exploitation de manière plus pragmatique et stratégique en s'appuyant sur les matériaux culturels (Rindova, Dalpiaz et Ravasi, 2011). Cette nouvelle vision culturelle nous a aidé à éclaircir de plus en plus notre vision sur l'innovation et de l'entrepreneuriat. Ainsi, elle a mis en relief le rôle primordial de la culture dans le redéploiement des nouveaux aspects technologiques et la création de nouvelles entreprises. Cette vision est omniprésente dans les approches populaires de l'entrepreneuriat et de l'innovation et basé sur les travaux de Hofstede (1998).

En plus, des multiples recherches ont mis en exergue la relation entre l'entrepreneuriat et l'innovation selon la croissance économique (Naudé ; 2008) et les pays en développement Szirmai et al. (2011). D'ailleurs, l'effet de l'activité entrepreneuriale sur la croissance économique est plus élevé dans les pays développés que dans les pays en voie de développement (Van Stel et al;2005).

\section{Materiel \& Methodologie}

Une étude documentaire selon une approche descriptive a été réalisée par le biais d'une analyse des rapports, des monographies et des travaux scientifiques portant sur ledit sujet ainsi que l'interprétation des divers schémas et diagrammes en prenant comme cas d'étude la société Nationale de Radiodiffusion et de Télévision (SNRT).

Or, l'analyse documentaire a été effectuée via les rapports collectés auprès du haut-commissariat au plan, la haute autorité de la communication audiovisuelle (HACA), le centre cinématographique, le ministère de la culture, de la jeunesse et des sports, la cour des comptes, le ministère de l'économie et des finances.

L'objectif de cette étude est d'analyser les indicateurs de mesure de l'impact de l'entrepreneuriat innovant sur le secteur audiovisuel au Maroc. Pour cela, nous avons procédé à une analyse quantitative ayant visée à extraire des déductions claires sur la base d'indications chiffrées. Cependant, l'analyse effectuée des différents concepts lors de la partie théorique, nous a permis de cerner notre problématique et décortiquer la relation entre l'entrepreneuriat innovant et le secteur audiovisuel. Ainsi, nous avons formulé les deux hypothèses mentionnées ci-après: 
Hypothèse 1: l'entrepreneuriat innovant est un vecteur vital pour relancer le secteur audiovisuel au Maroc; Hypothèse 2: l'entrepreneuriat innovant a un impact négatif sur le secteur audiovisuel au Maroc.

\section{ANALYSE ET DISCUSSION DES RESULTATS}

\section{A. Analyse des résultats}

Selon ce tableau, nous avons constaté que le budget destiné aux différentes catégories a été diminué lors de l'année 2019 jusqu'à l'année 2020. En effet, une variation de -77,49\% est constatée pour le long métrage, une nette diminution de $98,98 \%$ pour le film institutionnel et une absence remarquable de budget pour les téléfilms. Le taux a connu une nette chute de $73,50 \%$ dans la totalité.

Tableau 1 Budgets alloués à l'investissement par catégories

\begin{tabular}{|c|c|c|c|c|}
\hline \multirow{2}{*}{ Catégorie } & Année 2019 & Année 2020 & \multirow{2}{*}{\multicolumn{2}{|c|}{ Variation en $\%$}} \\
\hline & \multicolumn{2}{|c|}{ Budget en DH HT } & & \\
\hline Long métrage & $265.304 .959,15$ & $59.728 .908,00$ & - 77,49 & \\
\hline Court métrage & $1.214 .033,54$ & $493.777,77$ & $-59,33$ & \\
\hline Spot publicitaire & $14.850 .005,51$ & $13.171 .107,88$ & $-11,31$ & \\
\hline Série télévisée & $504.140 .624,95$ & $130.128 .729,30$ & $-74,19$ & \\
\hline Clip & $7.195 .192,29$ & $2.056 .652,91$ & $-71,42$ & \\
\hline Film institurionnel & $1.969 .279,43$ & $20.000,00$ & $-98,98$ & \\
\hline Téléfilms & $1.584 .895,22$ & - & $-100,00$ & \\
\hline Docu-fictions & $228.174,60$ & $5.430 .565,00$ & $+2.280,00$ & \\
\hline Total & $796.487 .164,69$ & $211.029 .740,86$ & $-73,50$ & \\
\hline
\end{tabular}

D'après ce schéma, le budget dans le secteur audiovisuel investi par les étrangers au Maroc a passé de plus de $720000 \mathrm{DH}$ en 2018 au $800000 \mathrm{DH}$ en 2019. Ce montant a diminué en 2020, le chiffre a passé à 200000 DH. Nous pouvons déduire que cette constatation est due à l'effet de la crise sanitaire Covid 19. 
Figure 1. Evolution des montants étrangers investis au Maroc

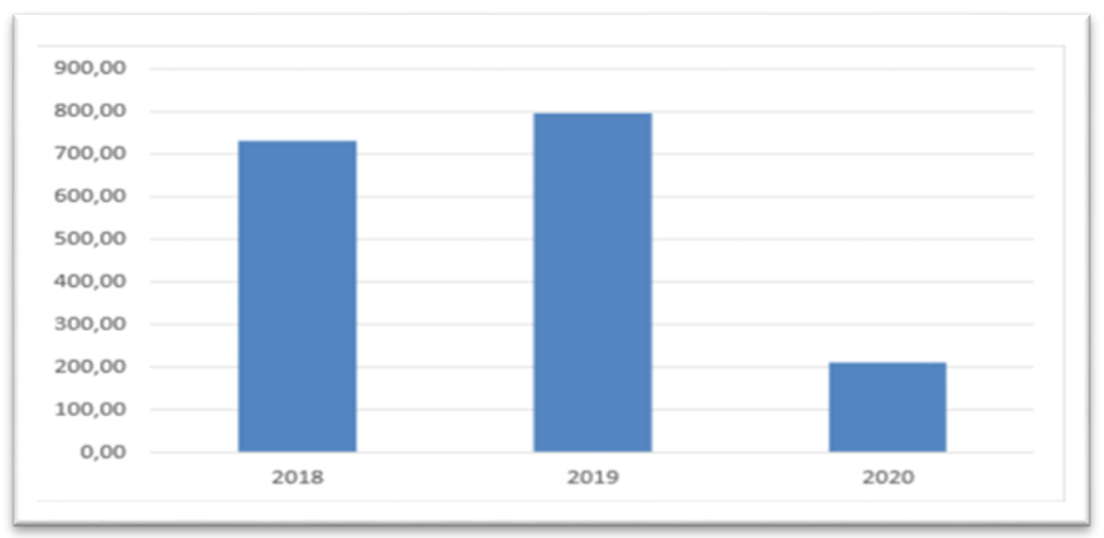

Source: Centre cinématographique 2020

Le capital humain est un élément clé de la réussite d'une telle stratégie. Selon ce diagramme, nous remarquons une fluctuation selon le sexe et les tranches d'âge. Entre 25 ans à 29 ans, les femmes ont un taux de $12 \%$ tandis que les hommes ont un taux de 10\%. Par contre, de 34 ans aux 44 ans, ce sont les taux les plus élevés pour les deux sexes, ils sont plus que $20 \%$. Il s'est avéré que l'élaboration d'une stratégie prévisionnelle de gestion de ressources humaines soit impérative.

Figure 2. Pyramide des âges au sein de SNRT

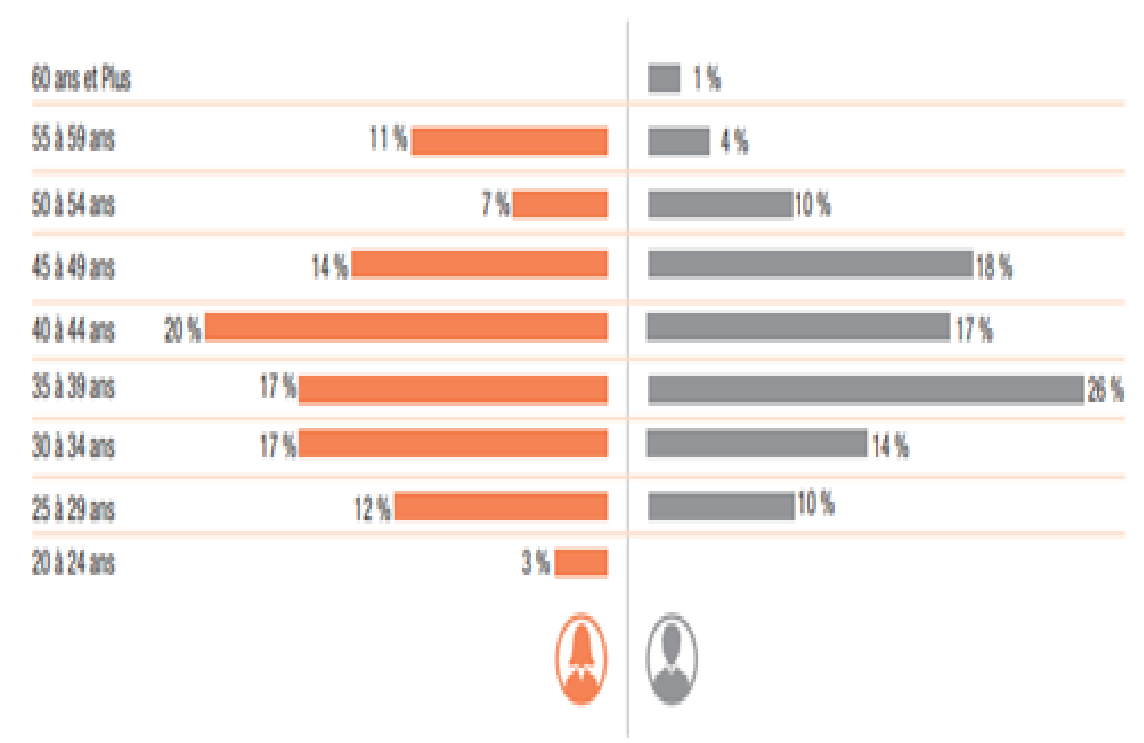

Source: HACA 2019

En outre, consciente de l'importance de la formation continue dans le développement des compétences de ses personnels, la mise à jour de leur acquis et le suivi de rythme de développement, la société (SNRT) a mis en œuvre un programme ambitieux. En effet, 50\% du personnel s'est orienté vers la formation de métier de régulation d'audiovisuel, tandis que le reliquat s'intéresse de plus à la formation de la langue, au management et de l'informatique. 
Figure 3. Répartition des formations continues réalisées

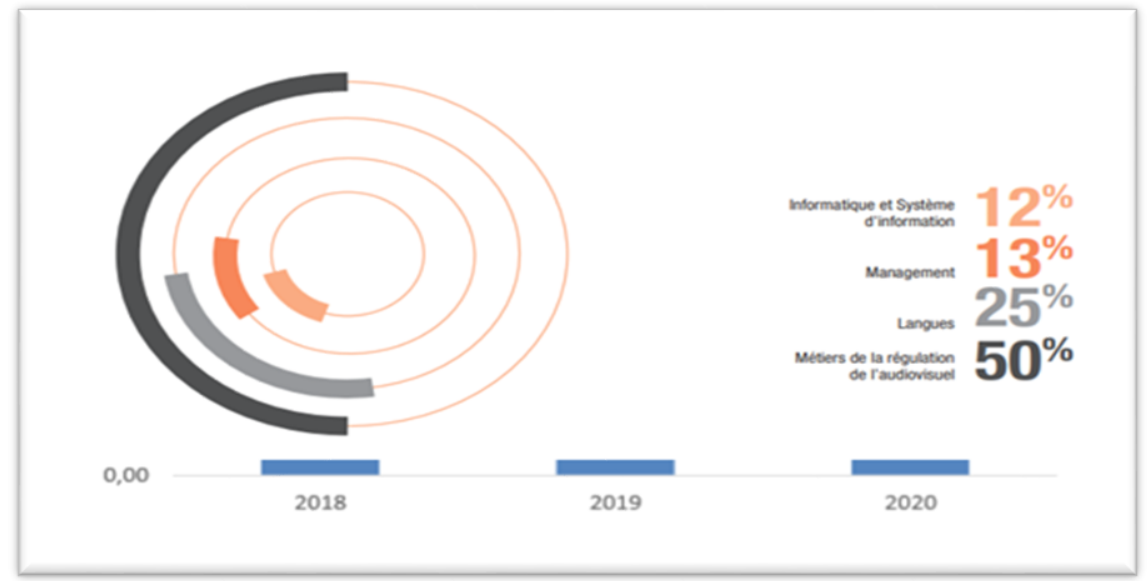

Source: HACA 2019

Le schéma suivant démontre que toutes les catégories professionnelles sont censées à effectuer des formations diversifiées selon leur domaine d'activité. 64\% constitue la part des stagiaires, $18 \%$ représente les techniciens et $12 \%, 4 \%$ et $2 \%$ correspondent respectivement aux catégories professionnelles : les directeurs du service, les directeurs de division et les cadres.

Figure 4. Répartition des formations selon les catégories professionnelles

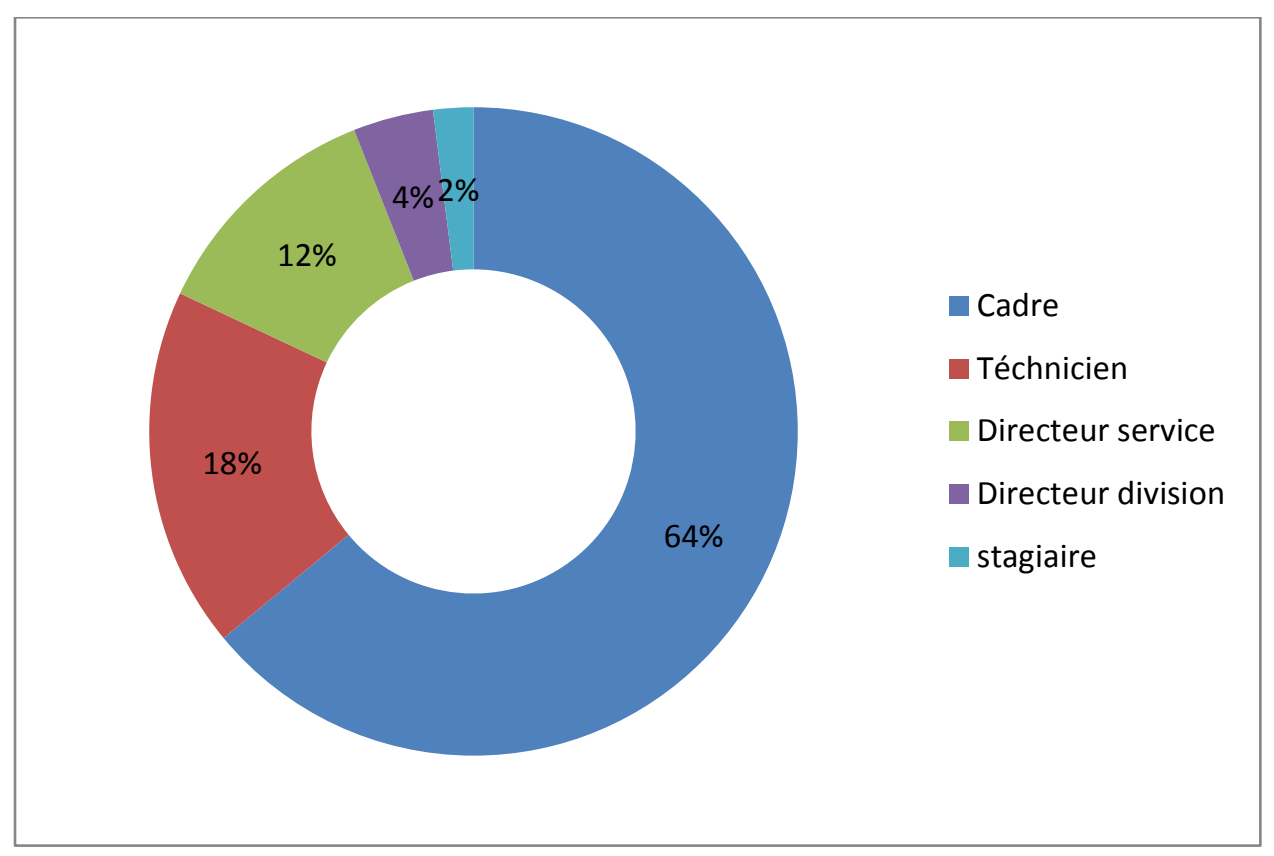

Source: Etabli par nos propres soins

L'illustration mentionnée ci-dessous a démontré que l'audience moyenne des chaînes nationales locales a passé de 41,43\% à 51,5\% depuis 2013 puis à 2019. Cependant, les taux des autres pays arabes à l'instar du Kuwait 8,8\%, Oman 14,5\%, Qatar 6,4\%, l'Arabie Saoudite 6,0\% et les Emirates arabes Unies 8,0\% restent très modestes. 
Figure 5. Audience moyenne des chaînes nationales et arabes

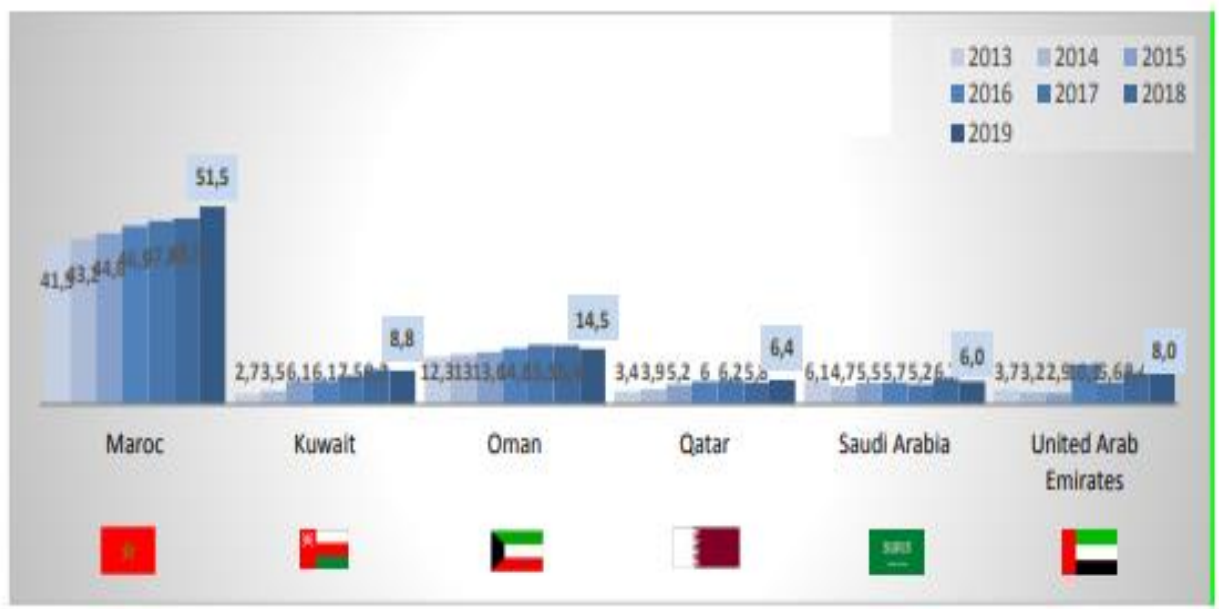

Source: HACA 2019

Le diagramme ci-après a mis en évidence l'origine des parts financières de soutien de ce secteur dans les quinze dernières années. L'Etat marocain contribue de plus de 345 en 2006. Ce chiffre a passé à 1150 en 2020. Toutefois le reste n'a pas dépassé en 2020, 129 et 206 respectivement pour la caisse de fonds de développement de secteur audiovisuel et l'impôt collecté pour ce secteur.

Figure 6. Parts de soutien financier destinés au secteur audiovisuel

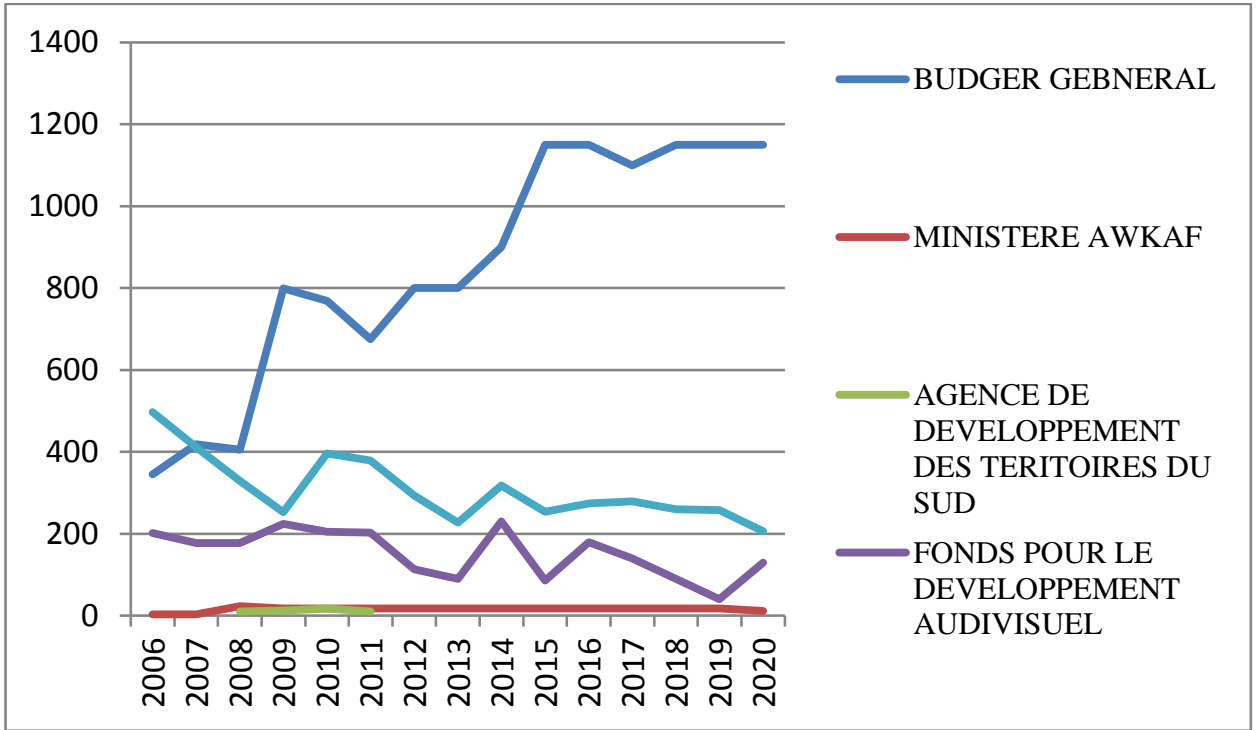

Source: Etabli par nos propres soins

Le volume des recettes des opérateurs marocains par les activités de ce secteur est diversifié. Les revenus en million de dirhams de la publicité ont connu une fluctuation depuis 2006 jusqu'à 2020, ils ont été passés de 153 à 117 millions de dirhams. En revanche, les revenues de droit sportif ont été 22 millions en 2007, ils ont passé à 156 millions en 2020. Cette diminution dépend de l'effet remarquable de la crise sanitaire sur l'économie nationale. 
Figure 7. Diverses recettes de secteur audiovisuel

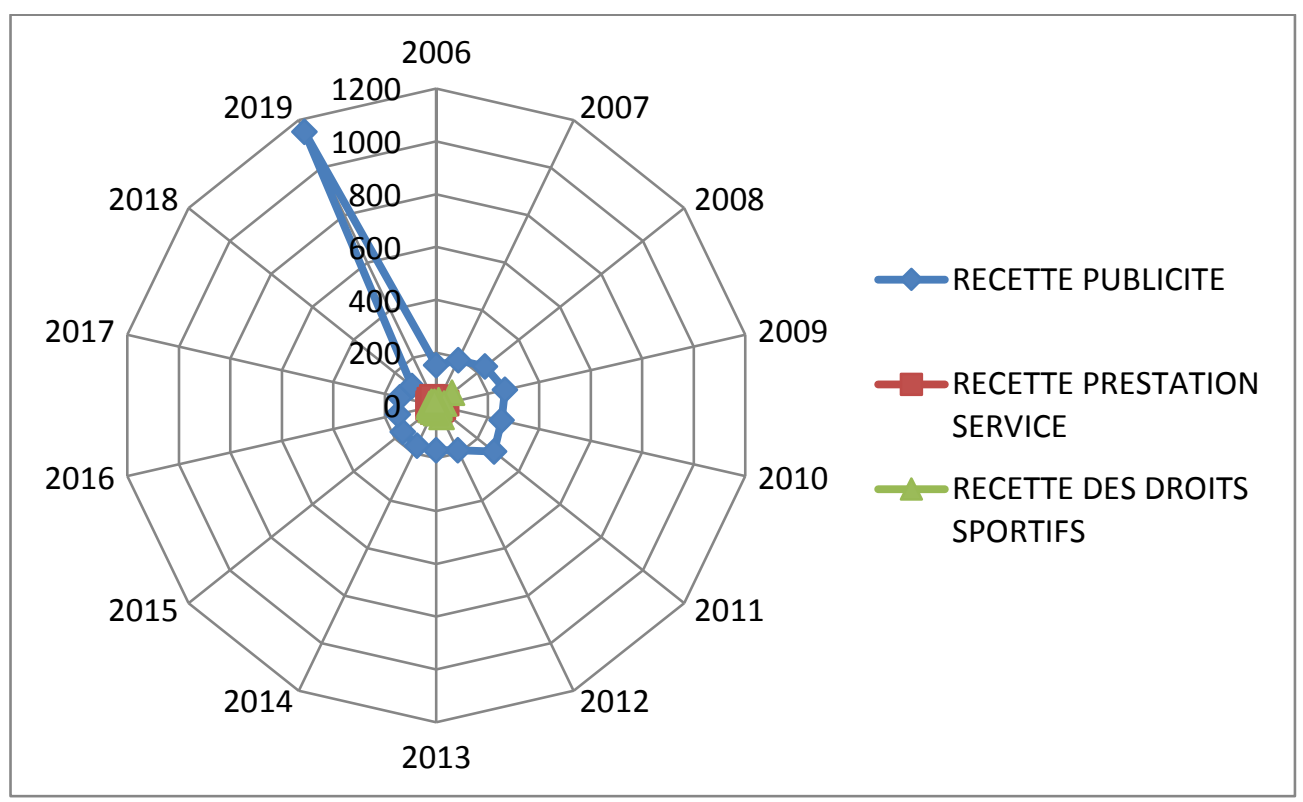

Source: Etabli par nos propres soins

La diversité et l'évolution de la qualité des produits destinés à la clientèle ont impacté les investissements au sein de la SNRT au Maroc. Le total de l'investissement de 2002 à 2005 a été 595 millions de dirhams. Or, depuis 2006 à 2020 la valeur a connu une augmentation de plus de 1700 millions.

Figure 8. Investissement de SNRT

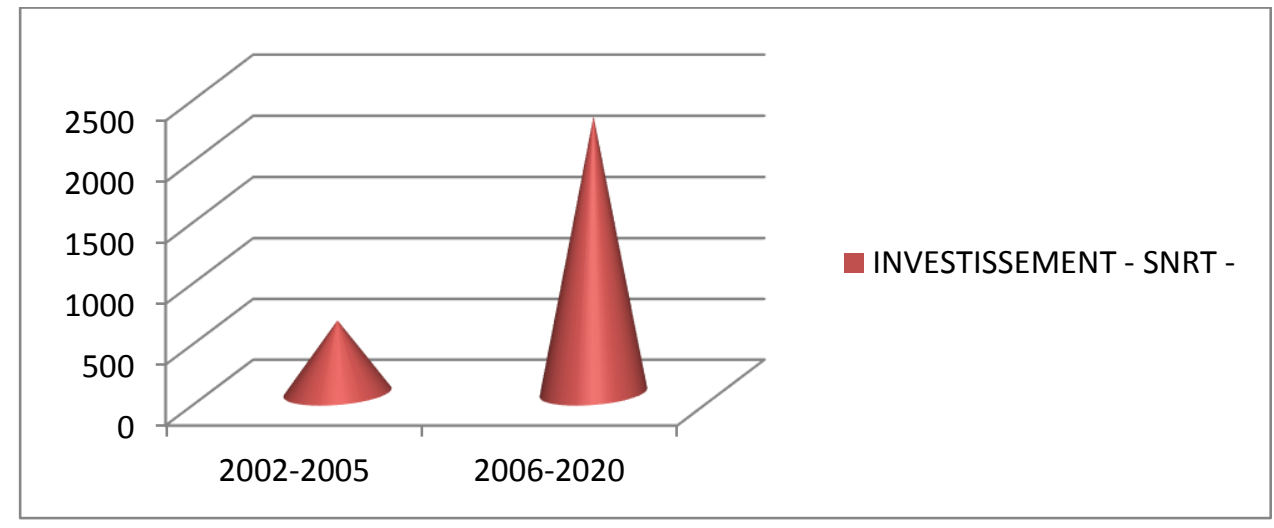

Source: Etabli par nos propres soins

Nous sommes basés sur cette présentation graphique pour visualiser le circuit d'achat établi au sein de la société Nationale de Radiodiffusion et de Télévision. L'étude de ce processus nous a permis d'avoir une vision plus claire. Il s'agit d'un système caractérisé par la transparence et la crédibilité ayant mis en œuvre durant toutes les étapes d'approvisionnement notamment, la détermination des cahiers de charges, la publication des appels d'offres et le traitement et la validation des dossiers. Ce climat incite de plus en plus les acteurs privés à investir dans le domaine audiovisuel. 
Figure 9. Processus d'achat au sein de SNRT -2021-

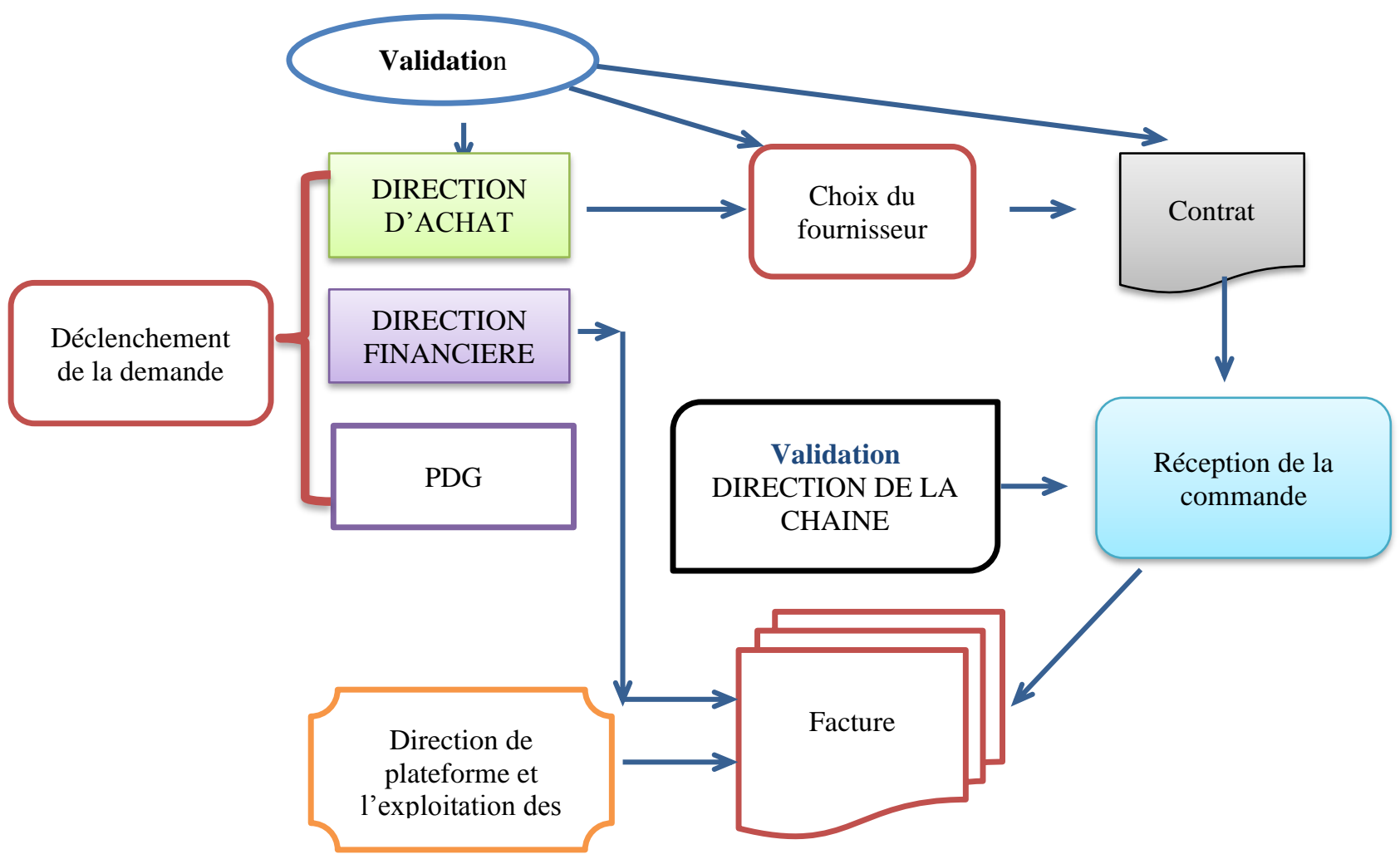

Source : Etabli par nos propres soins

\section{B. Discussion des résultats}

L'analyse des données recueillies et les interprétations de diverses configurations statistiques ainsi que la lecture des notions théorique, des rapports et des recherches établis dans ce sens nous a permis de constituer une idée globale sur notre sujet. Notre première hypothèse a été bien confirmée. En effet, l'entrepreneuriat est en corrélation positive avec le développement de secteur d'audiovisuel au Maroc. Tandis que, nous avons conclu que notre deuxième hypothèse a été complètement infirmée. Du coup, l'entrepreneuriat a un impact positif à court terme sur ce secteur. Alors que, vu le coût élevé de la mise en exécution des programmes et des actions innovantes et créatives, l'impact de l'innovation ne sera concrétisé effectivement qu'après une période déterminée.

Par ailleurs, les autorités marocaines ont défini une stratégie audiovisuelle prometteuse. Elles ont mis en place une panoplie de ressources d'ordre matériel et immatériel pour encourager l'entrepreneuriat innovant artistique. Par conséquent, elles ont pu créer un environnement dynamique et résister à la concurrence privée et étrangère. En effet, les chaines ayant constitué le bouquet de la SNRT, Sored 2M et Medi 1 TV, les radios ainsi que les chaines électroniques diffusent leurs programmes via les modes techniques analogiques ou numériques et réussissent à garantir des volumes de recettes importantes. La convergence technologique et le développement des systèmes informatiques dans le secteur audiovisuel est un objectif essentiel escompté afin d'innover le processus médiatique marocain. Pour cela, par le biais de l'intégration de nouveaux modes plus sophistiqués de la réception et le perfectionnement de système d'enregistrement, le monitoring des programmes (HACA Media Solutions) s'est revêtu d'une importance cruciale. En outre, une plateforme a été 
dédiée aux différents opérateurs surnommée (HACA Bridge) et caractérisée par un système développé sécurisé (Télé-déclaration, consultation et notification).

Grâce à l'innovation technologique et leur esprit entrepreneurial, les responsables marocains ont investi dans la couverture des manifestations culturelles, sportives et socioéconomiques en ajoutant IP aux supports de transmission satellite et les faisceaux hertziens ainsi aux enregistrements et l'exploitation des signaux. En plus, la résolution des images a passé de SD (Définition standard de la résolution 480p)au HD (Haute Définition standard de la résolution 1080p ou de 720p). Cette nouvelle technologie contribue au développement de la potentialité de ce secteur.

En outre, le Conseil Supérieur de la Communication Audiovisuelle a insisté sur le respect des droits des hommes à travers des programmes télévisuels ayant le but d'éviter toutes sortes de discrimination entre les sexes et les personnes en situation de handicap. En plus, chaque intervenant a le droit de présenter ses idées malgré la différenciation des orientations politiques et idéologiques dans un climat de pluralisme.

Par ailleurs, grâce à la digitalisation, le secteur a connu une extension remarquable. Ce développement numérique permet à SNRT de mettre en place un nouveau système appelé 'Trafic management'. Il s'agit des plateformes à l'instar de :

SNRTLIVE : l'utilisateur peut accéder à l'information via son téléphone par le biais de la technologie Shifting-time ;

BOTOLA : permet de consulter les dernières informations sportives et regarder des matchs via une application mobile ;

YOUTUBE SNRT : est une chaine dédiée aux utilisateurs des réseaux sociaux et d'internet;

DEMAND ON VIDEO-VOD : consiste à répondre à la demande via le deuxième écran numérique (SCREEN SECOND).

Or, le Maroc, engagé dans le processus de la démocratie et la modernisation, a mis en exécution une plateforme médiatique. Il vise à répondre aux besoins du citoyen dans un monde compétitif où l'accès à l'information se fait aisément et immédiatement.

En dépit de la récession économique révélée lors de la pandémie Covid 19 et son impact sur l'économie nationale et internationale, le secteur audiovisuel a manifesté une résistance remarquable. En plus, des actions créatives et plus compétitives ont été préétablies par le nouveau modèle de développement présenté par la commission spéciale sur le modèle de développement (CSMD).

A cet effet, un diagnostic a été effectué afin d'appréhender les différentes lacunes et décortiquer les potentialités de ce secteur. Ainsi, un modèle selon une approche multidimensionnelle axée sur la bonne gouvernance, une gestion organisationnelle plus performante, des normes de transparence et le respect des valeurs de la nation, a été envisagé à le mettre à l'exécution à court et moyen termes. Pour conclure, l'entrepreneuriat innovant est indispensable afin de relancer le secteur audiovisuel, le développer, et le rendre plus compétitif.

\section{CONCLUSION}

Lors de ce travail, nous avons mis en relief l'impact de l'entrepreneuriat innovant sur le développement du secteur audiovisuel au Maroc. A cet égard, nous avons adopté une approche documentaire descriptive. Dans 
un premier lieu, l'étude a été axée sur une analyse de cadre théorique. Il s'agit, ainsi, de situer notre sujet selon les citations des économistes, les recherches et les travaux effectués. Ensuite, la lecture des rapports, les essais scientifiques et académiques, des divers tableaux, des schémas et des indicateurs nous a permis de constituer une vision plus exhaustive et bien précise.

La phase de la discussion des résultats était pour nous une étape déterminante. Du coup, nous avons pu confirmer notre hypothèse notamment l'entrepreneuriat innovant est un vecteur vital ayant un impact positif sur la relance et le développement de secteur de l'audiovisuel au Maroc. Tandis que cette étude nous a infirmé notre deuxième hypothèse.

Or, l'Etat marocain, à l'instar de plusieurs pays émergents, a instauré une série de mesures capables d'assurer un environnement idoine afin d'encourager l'entrepreneuriat et inciter toutes les initiatives d'innovation. D'ailleurs, un des axes du nouveau modèle de développement a précisé et confirmé la volonté du gouvernement marocain à libérer et soutenir toutes les actions entrepreneuriales innovantes.

Néanmoins, des multiples entraves matérielles et immatérielles peuvent ralentir la concrétisation de la volonté de l'Etat pour atteindre ses objectifs consentis. Une action corrective est impérative afin de concevoir chaque défaillance et d'intervenir au moment opportun.

Par ailleurs, lors de cette recherche l'analyse a été centrée sur une approche documentaire et descriptive. La multiplication et la diversité des informations ainsi que des études qualitatives et quantitatives peuvent détailler de plus cette recherche et confirmer nos résultats.

En outre, l'intégration des paramètres modérateurs comme le rôle du capital humain dans ce processus ainsi que le secteur privé comme une partie importante peuvent être une base d'étude lors des prochaines recherches académiques.

\section{BIBLIOGRAPHIE}

[1] A. Baregheh , J. Rowley, and S. Bangor, Towards a multidisciplinary definition of innovation.

[2] A. Szitmai, W. Naudé, and M. Goedhuys, Entrepreneurship,Innovation, and Economic Development, Oxford University Press,2011.

[3] A.Brem, "Linking innovation and entrepreneurship: literature overview and introduction of a process-oriented framework", International Journal of Entrepreneurship and Innovation Management, Vol. 14 No. 1, pp. 6-35,2011.

[4] A.Van Stel, M.Carree and R. Thurik, “The effect of entrepreneurial activity on national economic growth", Small Business Economics, 24(3), pp.311-321,2005.

[5] B.González? and P.A.Muñoz-Gallego, " Role of entrepreneurship and market orientation in firms success", European Journal of Marketing, 43(3/4), pp.500-522, 2009.

[6] C.Feki and S.Mnif, “Entrepreneurship, Technological Innovation, and Economic Growth: Empirical Analysis of Panel Data, Journal of the Knowledge Economy,Vol. 7, pp.984-999, 2016;

[7] C.Fernandes, J. Ferreira, and C.S.Marques, Innovation management capabilities in rural and urban knowledge intensive business services: empirical evidence, Springer-Verlag Berlin Heidelberg, 2013.

[8] C.Marcotte, "Entrepreneurship and innovation in emerging economies: Conceptual, methodological and contextual issues", International Journal of Entrepreneurial Behavior \& Research, Vol. 20, No. 1, pp. 42-65,2014.

[9] C.Rodica, "The role of innovative entrepreneurship in the economic development of EU member countries Journal of Entrepreneurship, Management and Innovati on (JEMI), Vol. 15, Issue.1,pp.35-6,2019.

[10] Centre cinématographique marocain https://www.ccm.ma/actualite-1849

[11] D. Smallbone, and F.Welter (2010), "Entrepreneurship and institutional change in transition economies: a discussion paper", Seminar at St Petersburg State University, St Petersburg, May, available at: www.gsom.spbu.ru/files/upload/niim/seminar/smallbone_discussion_paper.pdf (accessed June 20, 2012). 
[12] D. Smallbone, and F.Welter ,"Entrepreneurship and institutional change in transition economies: the commonwealth of independent states, Central and Eastern Europe and China compared", Entrepreneurship and Regional Development, Vol. 24 ,No. 3-4, pp. 215-233,2010.

[13] D.Doloreux, Y.Melancon , “Onthe dynamics of innovation in Quebec's coastal maritime industry”, Technovation, 28,pp.231243,2008 .

[14] D.Ian. S.Parkman, S. Holloway, and S.Helder, "Creative industries: aligning entrepreneurial orientation and innovation capacit', Journal of Research in Marketing and Entrepreneurship,Vol. 14, Iss 1, pp. 95 - 114,2012.

[15] D.Miller,“The correlates of entrepreneurship in three types of firms”,Management Science, Vol. 29, pp. 770-91,1983.

[16] D.Ribeiro, Soriano, and A.Kun-Huang, “Innovation and entrepreneurship in knowledge industries”, Journal of Business Research ,Vol. 66, Issue .10, pp. 1964-1969, Oct, 2013.

[17] D.V.Brazeal, and T.T.Herbert,“The genesis of entrepreneurship ”, Entrepreneurship Theory and Practice, Vol. 23, No. 3, pp. 29-45,1999.

[18] E.Dalpiaz,V.Rindova, and D.Ravasi , "Combining logics to transform organizational agency blending industry and art at Alessi”, Administrative Science Quarterly, Vol.61, pp.347-392,2016.

[19] F.Zhao "Exploring the synergy between entrepreneurship and innovation”, International Journal of Entrepreneurial Behaviour \& Research, Vol. 11 ,No. 1, pp. 25-41, 2005.

[20] G Hofstede , "Attitudes Values and Organizational Culture: Disentangling the Concepts ", Journals.sagepub.com,1998.

[21] G.Fisher, S.Kotha and A.Lahiri,“"Changing with the times: An integrated view of identity, legitimacy, and new venture life cycles ", Academy of Management Review, 41(3),pp.383-409,2016.

[22] G.Knight, "Cross-cultural reliability and validity of a scale to measure firm entrepreneurial orientation”, "Journal of Business Venturing", Vol. 12, pp. 213-25,1997.

[23] G.Xavier, “' Introduction: L'économie de la culture est-elle particulière ? ', Revue d'économie politique”, vol. 120, pp. 1-34, 2010.

[24] H.Erken, P.Donselaar, and R.Thurik, "Total factor productivity and the role of entrepreneurship", The Journal of Technology Transfer, 43(6), pp. 1493-1521, 2018.

[25] Haut-Commissariat au Plan https://www.hcp.ma/Etudes-economiques_r207.html

[26] Haute Autorité de la Communication :https://www.haca.ma/sites/default/files/upload/HACA\%20RAPrt\%202019\%20.pdf

Audiovisuelle

(HACA)

[27] J.Hu, Y, and Hsu Y, "The more interactive, the more innovative? A case study of South Korean cellular phone manufacturers", Technovation, 28(3), pp.75-87,2008.

[28] J.Schumpeter,The theory of economic development, Cambridge Mass: Harvard University Press, 1934.

[29] K.Ahouzi, and L. Nait Haddou, "L'entrepreneuriat innovant et le développement territorial : cas du secteur artisanal au Maroc", Contrôle de la comptabilité et de l'audit, Vol .03, pp. 864- 883, 2019.

[30] L.Iandoli, H.Landström, and M.Raffa, Entrepreneurship, Competitiveness and Local Development Frontiers in Entrepreneurship European Research,2005.

[31] M. Lounsbury, J.Cornelissen, N.Granqvist, and S,Grodal, Culture, innovation and entrepreneurship,2019, in DOI: $10.1080 / 14479338.2018 .1537716$

[32] M. Peng, "How entrepreneurs create wealth in transition economies", Academy of Management Executive, Vol. 15, No. 1, pp. 95-112, 2001.

[33] M.Fauzi, and T.D. Soehari, “"The effect of Entrepreneurial Attitude,Innovation and Creativity on Business success in the Garment Industry", Dinasti International Journal of Management Science, 2(1), pp.125-131 in https://doi.org/10.31933/dijms.v2i1.413, 2020.

[34] M.Kirzner, Competition and entrepreneurship. Chicago and London: University of Chicago Press,1973.

[35] M.Vivarelli, "is entrepreneurship necessarily good? Microeconomic evidence from developed and developing countries", Industrial and Corporate Change, 22(6), 1453-1495, 2013. 
[36] M.W. Peng, "Institutional transitions and strategic choices", Academy of Management Review, Vol. 28, No. 2, pp. 275-296, (2003).

[37] Ministère de Finance in https://www.ebu.ch/files/live/sites/ebu/files/Publications/euromed_MA_report.pdf

[38] P.Drucker, Innovation and Entrepreneurship. New York, NY,Harper Perennial,1985.

[39] P.M.Kreiser, L.D.Marino, and K.M.Weaver, "Assessing the psychometric properties of the entrepreneurial orientation scale: a multi-country analysis", Entrepreneurship Theory and Practice, Vol. 26, pp. 71-92,2002.

[40] P.Roberts and R.Amit (2003), The dynamics of innovative activity and competitive advantage: the case of Australian retail banking, 1981 to 1995. Organ Sci , 2003.

[41] P.Wong, Y.Ho and E.Autio, E, "Entrepreneurship, innovation and economic growth: Evidence from GEM data, Small Business Economics, 24(3), pp.335-350, 2005.

[42] R.Crudu, "The role of innovati ve entrepreneurship in the economic development of EU member countries", Journal of Entrepreneurship, Management and Innovati on (JEMI, Vol.15, Issue 1, pp.35-6, 2019.

[43] R.G .McGrath, I.C. MacMillan, E.A. Yang, and W. Tsai,"Does culture endure, or is it malleable? Issues for entrepreneurial economic development", Journal of Business Venturing, Vol. 7, pp. 441-58,1992.

[44] R.J.Arend, Entrepreneurship: A theory for no theory for now. Strategic Organization,2020 in doi:10.1177/1476127020979041.

[45] S. Aparicio, D. Urbano, and D. Audretsch, "Institutional factors, opportunity entrepreneurship and economic growth: Panel data evidence", Technological Forecasting \& Social Change, 102, pp.45-61, 2016.

[46] S.Gedeon, “What is Entrepreneurship?, ’Entrepreneurial pratice review', Vol.1, Issue. 3,2010.

[47] S.Hua, and U.Wemmerlov, “ Product change intensity, product advantage, and market performance: an empirical investigation of the PC industry, J Prod Innov Manage, 23, pp.316-329,2006.

[48] S.L. Mueller, and A.S. Thomas, "Culture and entrepreneurial potential: a nine-country study of locus of control and innovativeness", Journal of Business Venturing, Vol. 16 No. 1, pp. 51-75,2011.

[49] T.J.Eckhardt,A.Scott, and A. Shane, “Opportunities and Entrepreneurship', Journal of Management , 29(3) ,pp.333-349,2003.

[50] T.Koc, and C.Ceylan ,' Factors impacting the innovative capacity in large ,scale companies', Technovation, Vol. 27, Issue .3, pp .105-114,2007.

[51] T.Verstraete, “Entrepreneuriat : modélisation du phénomène'”, Revue de l'Entrepreneuriat', Vol. 1, pp. 5 - 23,2001.

[52] W. Bygrave, and C. Hofer, “Theorizing about entrepreneurship', Entrepreneurship Theory and Practice, Nr .16(2), pp.13-22, 1991

[53] W.Naudé, “Entrepreneurship in economic development”, Uni-Wider research paper, Nr. 2008/20. Helsinki: United Nations University, 2008.

[54] Y.Akinwale, J.Akinbami, and J. Akarakiri, "Factors influencing technology and innovation capability in the Nigerian indigenous oil firms", International Journal of Business Innovation and Research, pp.247-268, 2018 b. 